\title{
CONTROLE EM CASCATA DE UM MANIPULADOR ROBÓTICO COM UM ELO E UMA TRANSMISSÃO FLEXÍVEL
}

\author{
Alejandro Rafael Garcia Ramirez* \\ ramirez@das.ufsc.br
}

\author{
Edson R. de Pieri* \\ edson@das.ufsc.br
}

\author{
Raul Guenther* \\ guenther@emc.ufsc.br
}

* Laboratório de Robótica, Depto. de Automação e Sistemas, Universidade Federal de Santa Catarina P.O. Box 476, 88040-900 Florianópolis, SC, BRAZIL

\begin{abstract}
The position control of robot manipulators with joint flexibility is studied in this work. Two approaches are considered: one based on the property of Passivity and the Variable Structure Control, and another one based on the Backstepping technique and Passivity. The control strategies were tested in a robot prototype, specially built for this purpose. This work presents the experimental results which illustrates the applicability of the control methodologies.
\end{abstract}

KEYWORDS: Robot control, passivity, joint flexibility, cascade control, backstepping.

\section{RESUMO}

Este trabalho trata os problemas ocasionados pelas transmissões elásticas no controle de posição de robôs manipuladores. São apresentadas duas metodologias descritas na literatura: uma baseada na teoria da Passividade e o Controle a Estrutura Variável, e outra, que combina a técnica do Backstepping e a Passividade. Os experimentos realizados em um robô com transmissão flexível, construído para tal finalidade, mostram a validade das técnicas descritas no seguimento de trajetórias

Artigo submetido em $20 / 12 / 2000$

1a. Revisão em 20/2/2002; 2a. Revisão 17/9/2002;

3a. Revisão $24 / 10 / 2002$

Aceito sob recomendação do Ed. Assoc. Prof. Paulo E. Miyagi nesse tipo de robôs.

PALAVRAS-CHAVE: Robôs manipuladores, passividade, transmissão flexível, controle em cascata, integrador.

\section{INTRODUÇÃO}

Os robôs industriais são manipuladores formados por cadeias de corpos (elos), em cuja extremidade é fixada uma ferramenta ou dispositivo através do qual é realizada a tarefa. Os elos que formam a cadeia são interligados através de juntas que, de acordo com o movimento relativo entre os elos, podem ser de translação ou de rotação. O movimento da ferramenta é o resultado do movimento das juntas, realizado pelos atuadores e monitorado pelos sensores de posição e velocidade. Os atuadores podem ser elétricos, hidráulicos ou pneumáticos, sendo os elétricos os mais utilizados. Quando os atuadores são elétricos além do motor costuma ser necessária uma transmissão através da qual a velocidade do motor é adequada às necessidades do manipulador. Como o movimento da ferramenta é monitorado nas juntas, é comum que os elos sejam construídos de forma a serem bastante rígidos. O mesmo é desejável para os atuadores, em particular, que as transmissões sejam rígidas. Procura-se, desta forma, minimizar construtivamente os erros decorrentes da flexibilidade dos elos e das transmissões.

A flexibilidade intrínseca de algumas transmissões que 
utilizam harmonic drives e correias, por exemplo, limita esse procedimento e os robôs resultam com flexibilidade nas transmissões. Essa flexibilidade pode provocar erros de posicionamento, aumento no tempo de assentamento do robô, ressonância torsional e instabilidade. Desconsiderando a flexibilidade das transmissões mede-se, usualmente, a posição dos rotores. Por isso, os deslocamentos elásticos ocasionados pelas transmissões provocam erros no posicionamento em cada junta e, conseqüentemente, no posicionamento da ferramenta. Por outra parte, o tempo de assentamento dos sistemas dinâmicos depende diretamente de suas constantes de mola. Quanto mais flexível é o robô, maior é o seu tempo de assentamento. Outro fenômeno importante é ocasionado pela ressonância torsional. De fato, em sistemas mecânicos com movimentos de rotação, quando duas inércias são acopladas através de uma mola, esta pode armazenar uma grande quantidade de energia, o que pode provocar vibrações consideráveis se a freqüência de excitação é próxima da frequiência natural do sistema. Ainda, quando a flexibilidade das transmissões é desconsiderada, ela passa a constituir uma dinâmica não modelada, normalmente pouco amortecida, que, uma vez excitada pelo movimento do robô, pode provocar instabilidade. Esses efeitos refletem-se também no projeto dos controladores dos robôs limitando os ganhos de realimentação e, conseqüentemente, o desempenho. Isso foi verificado experimentalmente, ver por exemplo (Eppinger e Seering, 1992). Em (Spong e Vidyasagar, 1989) mostramse os erros de regime e o aumento do tempo de assentamento que ocorrem quando um robô com alguma flexibilidade nas transmissões é controlado por um algoritmo Proporcional-Derivativo (PD), medindo-se as posições e velocidades nos rotores. Em (Spong e Vidyasagar, 1989) mostra-se também que se as posições e velocidades para este caso forem medidas nos elos, o robô pode ser instável, mesmo para pequenos ganhos.

$\mathrm{Na}$ indústria, em função do tipo de tarefa que o robô realiza, o desempenho pode ser especificado em relação à velocidade de operação, precisão, carga que manipula, entre outros fatores. Em particular, quando o robô possui algum tipo de flexibilidade esses critérios de desempenho poderão não ser satisfeitos. De fato, é possível observar que muitos dos robôs industriais são projetados com a menor flexibilidade possível para satisfazer os diversos critérios estabelecidos usando controladores simples. Porém, existem aplicações nas quais a flexibilidade pode ser desejável. Por exemplo, nas aplicações industriais as limitações de peso levam ao projeto de estruturas mais leves que apresentam flexibilidade. Esta flexibilidade pode ser observada nos braços dos robôs e também nas transmissões devido à presença de harmonic drives, sensores de torque, entre outros tipos de acoplamentos elásticos. Também, nas aplicações que envolvem contato do robô com o meio, é necessário algum tipo de flexibilidade por motivos de segurança. Neste tipo de aplicações surgem problemas dinâmicos que podem levar à instabilidade. Isto se deve à presença dos chamados modos não colocados (o sensor e o atuador encontram-se em massas diferentes), o qual deve ser considerado na lei de controle (Eppinger e Seering, 1992). Em (Rocco et al., 1996) é apresentada uma lei de controle Proporcional-IntegralDerivativa (PID) no controle de força considerando uma transmissão flexível e se apresentam resultados experimentais. Nesse trabalho mostram-se as dificuldades que surgem quando a rigidez do contato é elevada. Em (Youcef-Toumi e Gutz, 1994) destaca-se a importância da dinâmica da transmissão flexível no momento do impacto de duas superfícies e trata-se o problema de controle. Em (Krzysztof e ElMaraghy, 1992) é proposta uma metodologia baseada na dinâmica inversa para o controle do contato robô com o meio rígido na presença de transmissões flexíveis.

Um material de estudo interessante surge nas aplicações espaciais (Albu-Schäffer e Hirzinger, 2000). Neste caso, os robôs são projetados visando maximizar a relação torque-massa e para isto, em muitos casos, são usados harmonic drives. Esses dispositivos, além de compactos, introduzem flexibilidade e atrito.

\subsection{Revisão bibliográfica}

Um robô com transmissões flexíveis tem o dobro de número de graus de liberdade e o mesmo número de atuadores que seu equivalente rígido. Trata-se de um sistema parcialmente acionado. Por isso os métodos usuais de controle de seguimento de trajetória, tais como: o controle baseado na dinâmica inversa, e o controle baseado em Lyapunov (DeWit et al., 1996) não podem ser aplicados diretamente.

A deterioração do desempenho dos robôs e as dificuldades teóricas introduzidas pela flexibilidade das transmissões motivaram um grande número de trabalhos de investigação e o desenvolvimento de controladores para robôs com transmissões flexíveis. No início as pesquisas desenvolveram-se em duas linhas. Uma empregando o enfoque da linearização por realimentação, numa tentativa de generalizar a técnica da dinâmica inversa, e outra utilizando o conceito de controle "composto", desenvolvido a partir do método das perturbações singulares, mediante o qual busca-se reduzir o número de graus de liberdade do robô com transmissões flexíveis ao número de graus de liberdade do robô rígido equivalente.

A linearização por realimentação foi desenvolvida a par- 
tir dos trabalhos de (De Luca et al., 1985; Spong e Vidyasagar, 1989; Nicosia e Tomei, 1988).Uma referência básica é Spong e Vidyasagar (1989). Uma dificuldade prática para a implementação desta técnica é a necessidade de medir as derivadas até de terceira ordem da posição do elo em relação ao tempo, no caso do modelo apresentar incertezas paramétricas.

No controle "composto"a redução do número de graus de liberdade é conseguida a partir da hipótese que a dinâmica adicional, ocasionada pela flexibilidade das transmissões, é suficientemente rápida para poder ser amortecida sem instabilizar a dinâmica principal, correspondente ao robô rígido. Assim, uma vez controlada a dinâmica adicional, o robô passa a ter seu comportamento como se fosse rígido. Com isso, os algoritmos de controle desenvolvidos para os robôs rígidos podem ser aproveitados também no caso das transmissões serem flexíveis. Este enfoque foi estudado por (Spong e Vidyasagar, 1989; Slotine e Li, 1991; Readman, 1994; Ghorbel e Spong, 2000), entre outros autores. A validade do enfoque é limitada aos casos em que a rigidez é suficientemente grande.

Com o objetivo de desenvolver controladores válidos para qualquer rigidez das transmissões e em que a derivada de terceira ordem da posição do elo em relação ao tempo não é necessária, surgiram, na década de 90 , diversas metodologias baseadas na estabilidade da conexão em cascata de sistemas estáveis (Seibert e Suarez, 1987). Destacam-se os trabalhos baseados na dinâmica inversa (Benallegue e M'Sirdi, 1990) e na passividade (Guenther e Hsu, 1993; Brogliato et al., 1995).

Com os mesmos objetivos em (Nicosia e Tomei, 1992) foi desenvolvido um controlador baseado na técnica do backstepping (Kokotovic, 1991). Essa técnica propõe uma metodologia sistemática que explora a presença de integradores no modelo do robô para a síntese da lei de controle. Uma dificuldade dos controladores desenvolvidos dessa forma é a superparametrização, que dificulta a implementação experimental da técnica, no caso do modelo apresentar incertezas paramétricas. Em (Fantoni e Lozano, 2000) apresenta-se um controlador robusto para evitar o excesso de parâmetros. Assim como os demais, Fantoni e Lozano (2000) apenas apresentam resultados de simulações.

Dos trabalhos examinados verifica-se que os diversos métodos propostos para o desenvolvimento de controladores para robôs com transmissões flexíveis não foram ainda validados experimentalmente.

Este trabalho pretende preencher essa lacuna apresentando a implementação experimental de duas estraté- gias baseadas na conexão em cascata. Ambas resultam em malhas fechadas globalmente estáveis, possibilitam aproveitar muitos resultados já obtidos para os robôs rígidos e não são limitadas no que diz respeito à rigidez das transmissões.

\section{MODELO DO ROBÔ}

A dinâmica do robô com juntas flexíveis pode ser descrita através do seguinte sistema de equações (Spong, 1987):

$$
\begin{gathered}
M\left(q_{1}\right) \ddot{q}_{1}+C\left(q_{1}, \dot{q}_{1}\right) \dot{q}_{1}+G\left(q_{1}\right)=K\left[q_{2}-q_{1}\right] \\
J \ddot{q}_{2}+K\left[q_{2}-q_{1}\right]=u,
\end{gathered}
$$

onde $q_{1} \in \mathcal{R}^{n}$ e $q_{2} \in \mathcal{R}^{n}$ representam as posições angulares dos elos e dos rotores, respectivamente; $\dot{q}_{1}$ é o vetor das velocidades angulares dos elos; $M\left(q_{1}\right)$, é a matriz de inércia do conjunto dos elos; $C\left(q_{1}, \dot{q}_{1}\right) \dot{q}_{1}$ é o vetor dos torques centrífugos e de Coriolis; $G\left(q_{1}\right)$ é o vetor dos torques gravitacionais; J é a matriz diagonal das inércias dos rotores; $K>0$ é uma matriz diagonal cujos elementos são os coeficientes de rigidez das transmissões; e $u$ é o vetor das forças generalizadas aplicadas nos rotores.

As equações do modelo (1) consistem de dois subsistemas dinâmicos acoplados, ver figura (1). Um representa o robô rígido tradicional, ou dinâmica dos elos, e outro, a dinâmica introduzida pela elasticidade das transmissões. Cabe observar ainda que o sistema é parcialmente acionado, pois os torques de controle são introduzidos apenas através dos rotores, existindo, portanto, $n$ torques de controle para $2 n$ graus de liberdade, onde $n$ é o número de elos do robô (Spong, 1987).

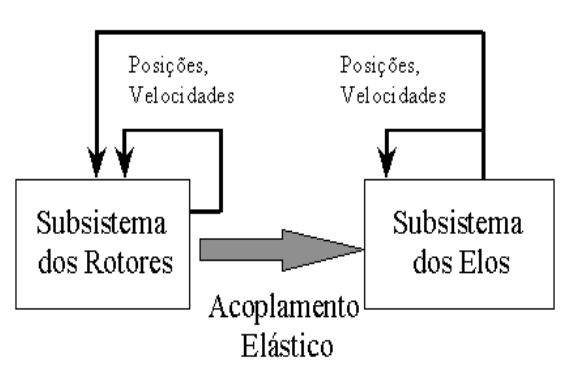

Figura 1: Modelo em cascata.

A elasticidade da transmissão é modelada como uma mola torsional linear. Esta simplificação é utilizada por diversos autores (ver, por exemplo, (Spong e Vidyasagar, 1989; Tomei, 1991; Readman, 1994; DeWit et al., 1996)). 


\subsection{Descrição do Robô}

O robô utilizado nos experimentos é um protótipo de um robô planar com dois elos, sendo que o acoplamento entre elos e motores pode ser rígido, ou flexível. Neste trabalho os experimentos foram realizados apenas no primeiro elo. A figura (2) ilustra os elementos que integram o robô.

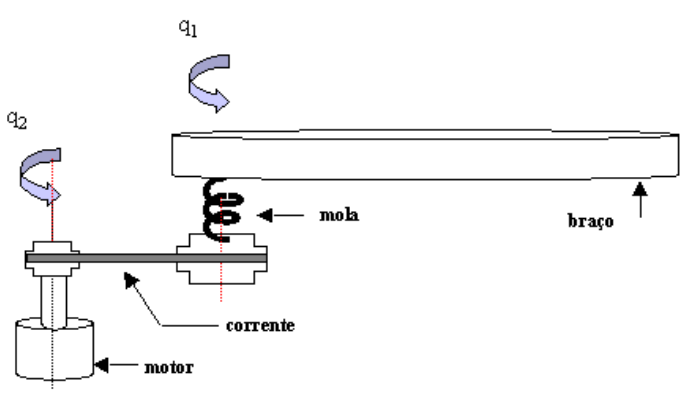

Figura 2: Elementos do robô.

Observar que o acoplamento entre o motor e o braço do robô é realizado através de uma transmissão de corrente e engrenagem, sendo a relação de redução $N=5$. A colocação, ou não, da mola implicará em um acoplamento flexível ou rígido entre o rotor e o elo, respectivamente.

Para o robô planar com apenas um elo as equações dinâmicas do modelo (1) ficam mais simples:

$$
\begin{gathered}
I \ddot{q}_{1}=K\left[q_{2}-q_{1}\right] \\
J \ddot{q}_{2}+K\left[q_{2}-q_{1}\right]=u,
\end{gathered}
$$

onde I é a inércia do conjunto elo mais a carga.

A seguinte tabela ilustra os parâmetros nominais do elo.

Tabela 1: Parâmetros nominais do elo.

\begin{tabular}{|c|c|}
\hline Parâmetros & Elo \\
\hline Inércia $\left(\mathrm{Kgm}^{2}\right)$ & 0.024 \\
\hline Massa $(\mathrm{Kg})$ & 3.179 \\
\hline Comprimento $(\mathrm{m})$ & 0.26 \\
\hline Centro de massa $(\mathrm{m})$ & 0.052 \\
\hline
\end{tabular}

Cabe ressaltar, que a inércia do elo foi medida em relação ao centro de massa, e o centro de massa do elo foi medido em relação ao eixo de rotação (Ramirez et al., 2002). A constante de mola da transmissão é de $6.77 \mathrm{Nm} / \mathrm{rad}$. A inércia do rotor é de $0.0052 \mathrm{kgm}^{2}$ e a constante de torque é de $0.25 \mathrm{Nm} / \mathrm{A}$.

\subsection{Modelo em cascata}

Através de uma mudança de coordenadas adequada pode ser transformado o sistema (2) em dois subsistemas em cascata com entradas $u$ e $q_{2 d}$. Esta mudança não é única (Brogliato et al., 1995).

A seguinte equação decompõe em cascata o sistema (2):

$$
u=J v+K\left[q_{2}-q_{1}\right]
$$

onde $v$ é uma função de controle aplicada no subsistema do rotor. Substituindo (3) em (2), obtém-se:

$$
\begin{gathered}
I \ddot{q}_{1}=u_{e d}+K \tilde{q}_{2} \\
\ddot{q}_{2}=v,
\end{gathered}
$$

onde $u_{e d}$ representa o torque aplicado na parcela rígida do robô e $\tilde{q_{2}}=q_{2}-q_{2 d}$ é o erro de seguimento no subsistema do rotor. O termo $q_{2 d}$ é a saída do subsistema do elo e pode ser interpretado como a posição de referência para o rotor de forma a garantir que o elo siga a trajetória desejada, como ilustra a equação (5).

$$
q_{2 d}=K^{-1}\left(u_{e d}\right)+q_{1}
$$

A decomposição em cascata (3) será usada na descrição leis de controle. Cabe ressaltar que, nas metodologias de controle baseadas nesta decomposição, deve ser garantida a continuidade e derivabilidade da trajetória de posição do elo de até ordem 4 , de forma a evitar descontinuidades na lei de controle.

\section{DESCRIÇÃO DO EXPERIMENTO}

O sistema flexível de desenvolvimento dSPACE (digital signal processing and control engineering) permite validar o projeto de controladores sobre um processo real diretamente a partir do diagrama de blocos implementado no Simulink/MATLAB. O projeto do controlador é compilado e executado em tempo real. A conexão com o robô é feita através de interfaces de entrada-saída. O sistema DS1102 é baseado na terceira geração do Processador Digital de Sinais, ou DSP (Digital Signal Processor) de ponto flutuante projetado pela Texas Instruments, e acrescida de um conjunto de periféricos freqüentemente utilizados em sistemas de controle digital. Basicamente, é composta por quatro conversores analógico para digital de 12 bits, ou ADC, quatro conversores digital para analógico de 12 bits, ou DAC, um microcontrolador DSP (TMS320C31) e dois codificadores incrementais que servem de interface aos sensores ópticos incrementais. A placa possui um conjunto de quatro portas digitais de entrada/saída de 16 bits, e três portas de 8 bits. 


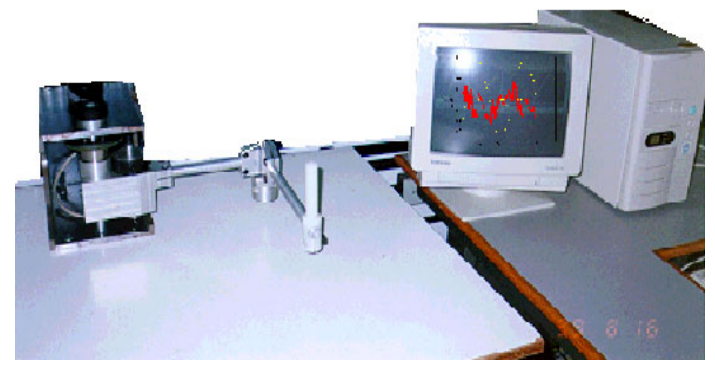

Figura 3: Instalação experimental utilizada nos testes de controle do robô com flexibilidade

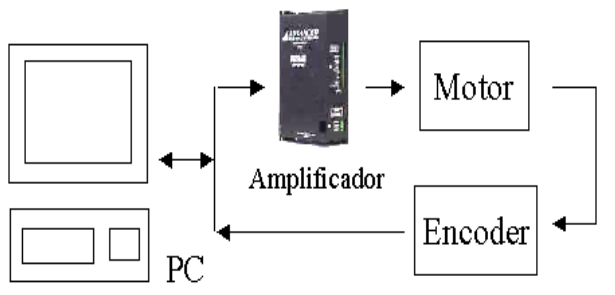

Figura 4: Principais elementos do sistema de controle.

\subsection{Instalação experimental}

A bancada experimental utilizada para implementação e testes dos controladores está instalada no LASHIP (Laboratório de Automação de Sistemas Hidráulicos e Pneumáticos) da UFSC e é mostrada na figura (3). A figura (4) ilustra os elementos que formam o sistema de controle entre o robô e a placa DSP.

A seguir são descritos esses elementos.

- Motor DCM8852 produzido por INLAND. Características: $1000 \mathrm{RPM}, 24 \mathrm{~V}$ de CC e $6 \mathrm{~A}$.

- Codificador angular HEDS-6010 da Hewlett Packard. Características: $56 \mathrm{~mm}$ de diâmetro, três canais, e 1024 linhas, que proporciona uma resolução de $0.0015 \mathrm{rad}$.

- Servo Amplificador modelo 12A8, produzido pela Advanced Motion Control. A corrente máxima de saída é de $12 \mathrm{~A}$. No modo de operação tensãocorrente (modo torque) a saída do amplificador é uma corrente proporcional à tensão na entrada.

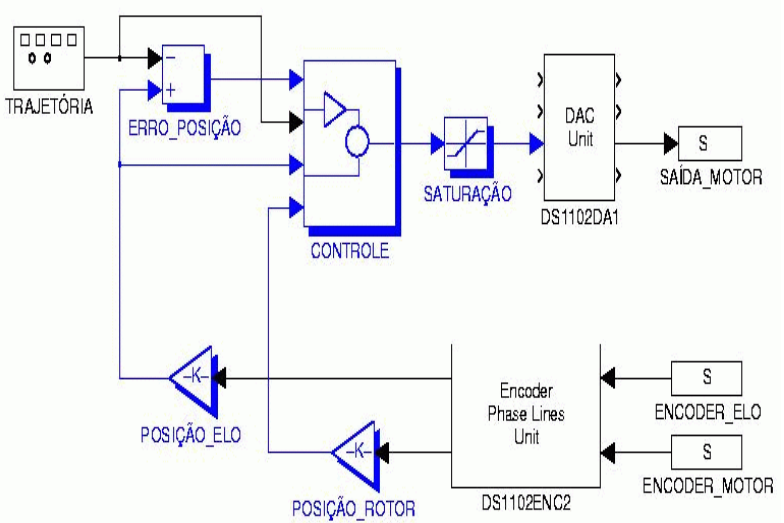

Figura 5: Estrutura de controle.

\subsection{Estrutura de Controle}

O controle é implementado seguindo a estrutura mostrada na figura (5).

Encoder-elo e Encoder-motor representam os sinais na saída dos codificadores incrementais solidários ao elo e ao eixo do motor, respectivamente. Posição-elo e Posição-rotor representam as transformações, em valores angulares de posição, das leituras disponibilizadas pelos codificadores incrementais na saída do bloco Encoder Phase Lines Unit.

Os movimentos do braço são projetados no bloco trajetória, enquanto o bloco Erro-Posição determina a diferença entre a posição desejada e a posição atual do elo a cada instante de tempo. No bloco controle é desenvolvida a estratégia de controle em cascata. Saída-motor é o sinal analógico resultante da ação de controle, o qual é enviado ao servo amplificador através do bloco de conversão Digital para Analógico (DAC). Cabe destacar que o motor foi controlado por meio da corrente de armadura usando o servo amplificador no modo torque. Os experimentos foram realizados usando-se um período de amostragem de $1 \mathrm{~ms}$.

\section{CONTROLE BASEADO NA PASSIVI- DADE}

O projeto é realizado em dois passos. O primeiro consiste em estabelecer a lei de controle para que o subsistema do elo siga a trajetória desejada. O segundo passo consiste em anular o erro de seguimento no rotor (Guenther, 1993). 


\subsection{Passo 1}

O torque aplicado na parcela rígida do robô utiliza o esquema baseado na passividade (Slotine e Li, 1991), sendo:

$$
u_{e d}=I \ddot{q_{1}}-K_{D 1} s_{1}
$$

onde $u_{e d}$ é o torque elástico desejado; $K_{D 1}$ é um ganho positivo de projeto, e:

$$
\tilde{q}_{1}=q_{1}-q_{1 d}, \dot{q_{r 1}}=\dot{q_{d 1}}-\lambda_{1} \tilde{q}_{1}, s_{1}=\dot{q_{1}}-\dot{q_{r 1}}
$$

onde $\lambda_{1}$ é um escalar positivo; $q_{r 1}$ é a velocidade de referência; e $s_{1}$ é interpretado como o erro de velocidade do elo.

A posição desejada para o rotor é:

$$
q_{2 d}=K^{-1}\left(u_{e d}\right)+q_{1}
$$

Substituindo (6) na primeira equação de (4), obtém-se a malha fechada deste subsistema:

$$
I \dot{s_{1}}+K_{D 1} s_{1}=K \tilde{q_{2}}
$$

onde $K \tilde{q_{2}}$ é considerado como uma pertubação à entrada deste subsistema. Quando $\tilde{q_{2}}$ é zero o sistema fica reduzido ao modelo rígido do robô manipulador, e é válida a propriedade da passividade (Slotine e Li, 1991).

\subsection{Passo 2}

Para o seguimento de trajetória no rotor é aplicado o torque de controle usando (3) (Guenther, 1993), resultando:

$$
u=J v_{0}+K\left[q_{2}-q_{1}\right]-K_{D 2} s_{2}
$$

onde $v_{0}$ é uma variável de controle nominal; $K_{D 2}$ é um ganho positivo; e $s_{2}$ é o erro auxiliar, formado por:

$$
s_{2}=\dot{\tilde{q}}{ }_{2}+\lambda_{2} \tilde{q_{2}}
$$

sendo $\tilde{q_{2}}=q_{2}-q_{2 d}$ é o erro de posição do rotor; e $\lambda_{2}$ é um escalar positivo. No caso ideal dos parâmetros conhecidos:

$$
v_{0}=\ddot{q_{2 d}}-\lambda_{2} \dot{\tilde{q_{2}}}
$$

Em (Guenther, 1993) mostra-se a estabilidade global da malha fechada formada pelas equações de seguimento dos elos e dos rotores mesmo quando os parâmetros do robô não são conhecidos. No mesmo trabalho mostra-se que a lei de controle descrita pode ser aplicada a robôs com mais graus de liberdade.

\section{CONTROLE BASEADO NO "BACKS- TEPPING"}

Consiste em resolver o problema de estabilizar o sistema em cascata, descrito na Equação (4), usando uma cadeia de integradores (Kokotovic, 1991). Nesta seção é descrita a metodologia apresentada em (Brogliato et al., 1995).

\subsection{Passo 1}

Utiliza-se também o esquema da passividade (Slotine e $\mathrm{Li}, 1991)$ de forma similar à primeira metodologia apresentada.

Definindo $q_{2}=q_{2 d}$ como a entrada de controle, sendo $q_{2 d}$ definido na Equação (8), a malha fechada no subsistema dos elos resulta:

$$
I \dot{s_{1}}+K_{D 1} s_{1}=0
$$

Este subsistema é passivo, mas quando $\tilde{q_{2}} \neq 0$ existe uma perturbação e a malha fechada fica similar a (9).

Adicionando-se um integrador à entrada deste subsistema, obtém-se:

$$
\dot{\tilde{q}}_{2}=\dot{q}_{2}-\dot{q}_{2 d}
$$

\subsection{Passo 2}

Assume-se que $\dot{q}_{2}=\dot{q}_{2 d}$ é a nova entrada de controle. Considera-se a seguinte função de Lyapunov para o sistema (9), (14) (Brogliato et al., 1995):

$$
V_{2}=V_{R}+\frac{1}{2} \tilde{q}_{2}^{T} \tilde{q}_{2}
$$

onde,

$$
V_{R}=\frac{1}{2} s_{1}^{T} I s_{1}+\tilde{q}_{1}^{T} \lambda_{1}^{T} K_{D 1} \tilde{q_{1}}
$$

$V_{R}$ é a função de energia da parcela passiva do robô (Slotine e Li, 1991).

Derivando $V_{2}$ em relação ao tempo, obtém-se:

$$
\dot{V}_{2}=\dot{V}_{R}+s_{1}^{T} K \tilde{q}_{2}+\tilde{q}_{2}^{T}\left[\dot{q}_{2}-\dot{q}_{2 d}\right]
$$

Uma escolha para $\dot{q}_{2}$ poderia ser (Brogliato et al., 1995):

$$
\dot{q}_{2}=-K s_{1}-\tilde{q}_{2}+\dot{q}_{2 d}
$$

Substituindo (17) em (16) cancela-se o termo $s_{1}^{T} K \tilde{q}_{2}$, e adiciona-se o termo quadrático $-\tilde{q}_{2}^{T} \tilde{q}_{2}$, o qual garante a estabilidade assintotica deste subsistema. 
Devido a que $\dot{q}_{2} \neq \dot{q}_{2 d}$ :

$$
\begin{gathered}
e_{2}=\dot{q}_{2}-e_{2 d} \\
e_{2 d}=-K s_{1}-\tilde{q_{2}}+q_{2 d}
\end{gathered}
$$

Observar que $e_{2}$ e $e_{2 d}$ não são as derivadas de $\tilde{q}_{2}$ e $q_{2 d}$, respectivamente.

Desta forma, obtém-se o seguinte sistema de equações em malha fechada:

$$
\begin{gathered}
I \dot{\dot{s}_{1}}+K_{D 1} s_{1}=K \tilde{q_{2}} \\
\dot{\tilde{q_{2}}}=e_{2}-K s_{1}-\tilde{q_{2}} \\
\dot{e_{2}}=-\dot{e}_{2 d}+v
\end{gathered}
$$

Observar que a última equação é equivalente a $\ddot{q_{2}}=v$. A presença do integrador originou a segunda equação do sistema (20).

\subsection{Passo 3}

Determina-se $v$ para que o sistema (20) seja globalmente assintoticamente estável. Para isso, define-se a seguinte função de Lyapunov:

$$
V_{3}=V_{2}+\frac{1}{2} e_{2}^{T} e_{2}
$$

Derivando $V_{3}$, obtém-se:

$$
\dot{V}_{3}=\dot{V}_{R}+s_{1}^{T} K \tilde{q}_{2}+\tilde{q}_{2}^{T}(\dot{\tilde{q}})+e_{2}^{T} \dot{e}_{2}
$$

Usando (20) e $v=-e_{2}+\dot{e_{2}}-\tilde{q_{2}}$, obtém-se:

$$
\dot{V}_{3}=\dot{V}_{R}-\dot{\tilde{q}}_{2}^{2}-e_{2}^{2}
$$

A Equação (22) garante a estabilidade global assintôtica da malha fechada (Brogliato et al., 1995).

Substituindo $v$ em (3) obtém-se a seguinte lei de controle:

$$
u=K\left[q_{2}-q_{1}\right]+J\left[\ddot{q_{2 d}}-2 \dot{\tilde{q_{2}}}-2 \tilde{q_{2}}-K \dot{s_{1}}-K s_{1}\right]
$$

Cabe ressaltar, que o backstepping é uma metodologia sistemática. Assim, é possível obter diferentes tipos de controladores realizando-se pequenas modificações nos passos do projeto (Nicosia e Tomei, 1992).

\section{RESULTADOS EXPERIMENTAIS}

Um dos principais resultados em relação à presença de transmissões flexíveis em robôs manipuladores, consiste no fato de que os controladores clássicos proporcional $(\mathrm{P})$, proporcional-derivativo $(\mathrm{PD})$ e proporcionalderivativo-integral (PID) não podem ser aplicados de forma direta neste tipo de robôs (Guenther, 1993). Na figura (6) observa-se o resultado da aplicação de uma lei de controle PD. A trajetória de posição, embora projetada para o elo, deve ser controlada nas coordenadas do rotor (posição angular) para evitar instabilidade (Spong e Vidyasagar, 1989).

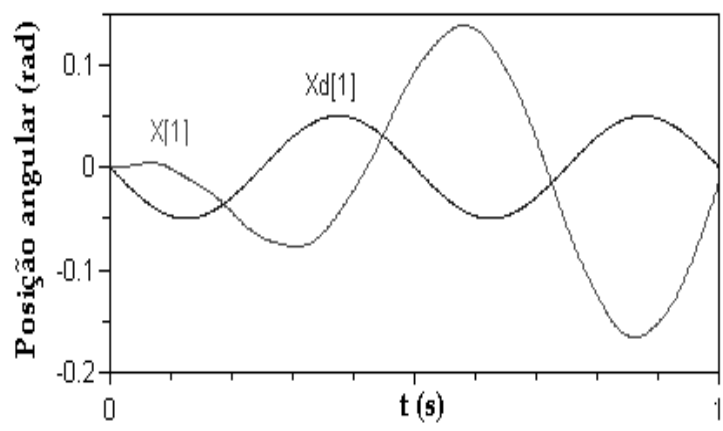

Figura 6: Controle PD: Trajetória do elo.

O resultado mostra que a posição do elo X[1] não consegue acompanhar a trajetória desejada $\mathrm{Xd}[1]$, como mostra a figura 6 .

A aplicação das metodologias de controle em cascata descritas neste trabalho resolvem o problema de controle observado na figura 6 .

\subsection{Controle baseado na passividade}

Na figura 7 mostra-se o desempenho do robô quando foi testada a metodologia de controle baseada na passividade e no controle a estrutura variável .

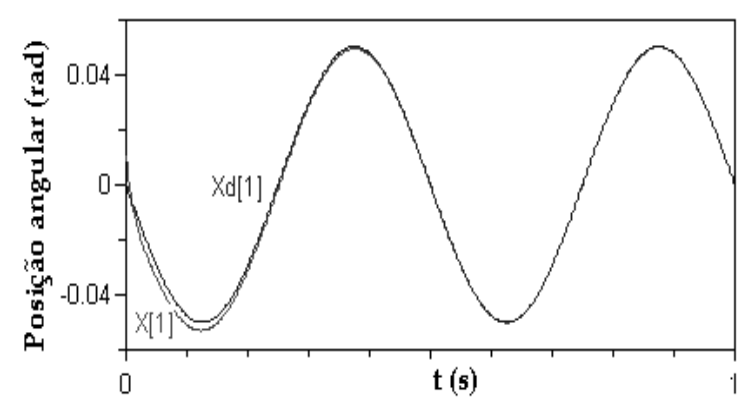

Figura 7: Trajetória do elo.

Observa-se o bom desempenho do controlador em relação ao seguimento de trajetória, pois a posição do elo $\mathrm{X}[1]$ segue a trajetória desejada $\mathrm{Xd}[1]$. 
Foi observa-da ainda a capacidade de rejeitar perturbações usando esta técnica de controle. A perturbação simula uma colisão aplicada diretamente no braço. Na figura 8 observa-se a rejeição à pertubação e os pequenos erros de seguimento obtidos em regime.

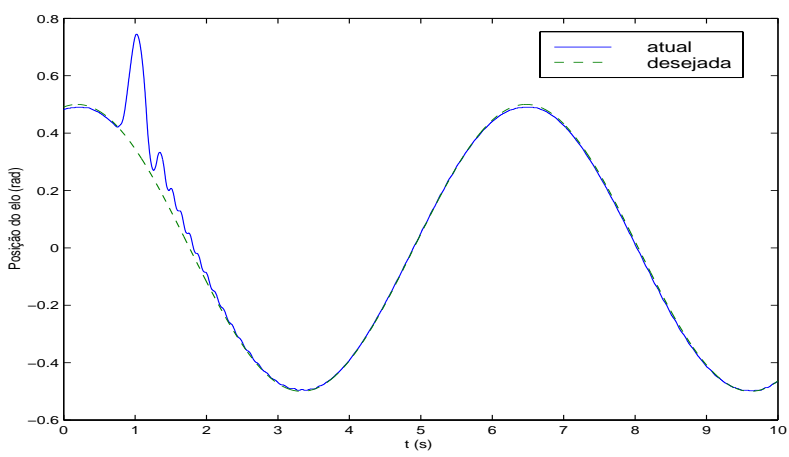

Figura 8: Colisão.

\subsection{Controle baseado no "backstepping"}

Nas figura 9 ilustra-se o desempenho do robô quando aplicada a metodologia de controle baseada no backstepping. Observa-se que a posição do elo X[1] segue a trajetória desejada $\mathrm{Xd}[1]$.

Na figura 10 foi simulada uma colisão aplicada diretamente no braço e foi observado o comportamento do robô. Observa-se a rejeição à pertubação e os pequenos erros de seguimento obtidos.

\section{CONCLUSÕES}

Neste trabalho foram apresentados os resultados experimentais obtidos em relação à aplicação de duas metodologias de controle em um robô planar com um elo e uma transmissão flexível. Cabe destacar que o projeto da bancada experimental envolveu desde a construção do robô, até o estudo e aplicação de uma placa de controle e aquisição de dados. Em relação aos resultados obtidos, cabe ressaltar que o desempenho do robô foi adequado mostrando-se a aplicabilidade de ambas as metodologias de controle. A ordem de complexidade das expressões de controle aplicadas foi similar, porém, na metodologia baseada no backstepping a complexidade aumentaria caso sejam adicionados outros passos de integração. Trabalhos futuros estão dirigidos à extensão destas metodologias ao segundo elo do robô. Outros aspectos a tratar seriam a implementação de outras técnicas de controle, considerando a compensação do atrito, e uma análise mais profunda em relação à presença de perturbações

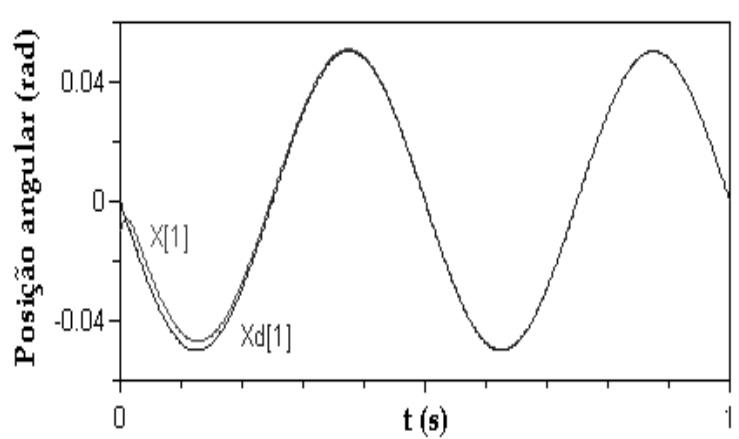

Figura 9: Trajetória do elo.

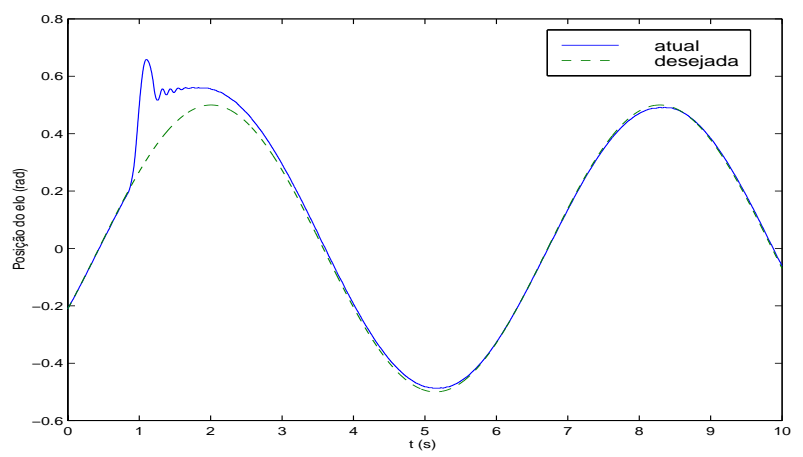

Figura 10: Colisão.

em malha aberta e malha fechada, diferentes daquela considerada neste trabalho.

\section{AGRADECIMENTOS}

Agradecemos à CAPES pelo suporte material e financeiro, o qual possibilitou a realização deste trabalho. Agradecemos também ao Engenheiro Roberto Kinceler pelo projeto do robô, e aos revisores pelas valiosas contribuições que permitiram melhorar o texto final.

\section{REFERÊNCIAS}

Albu-Schäffer, A. e Hirzinger, G. (2000). State feedback controller for flexible joint robots: A globally stable approach implemented on dlr's light-weight robots, In proc. IROS Japan.

Benallegue, M. e M'Sirdi, N. K. (1990). Control 
of robots manipulators with joint flexibility, Int. Workshop in Adaptive and Non Linear Control.

Brogliato, B., Ortega, R. e Lozano, R. (1995). Global tracking controllers for flexible-joint manipulators: a comparative study, Automatica 31(7): 941-956.

De Luca, A., Isidori, A. e Nicolò, F. (1985). Control of robot arm with elastics joints via nonlinear elastic feedback, 24th Conf. Decision and Control .

DeWit, C., Bruno Sciliano, B. e Georges, B. (1996). Teory of Robot Control, Springer - Verlag London Limited.

Eppinger, S. D. e Seering, W. P. (1992). Three dynamic problems in robot force control, IEEE Transactions on Robotics and Automation 8(6): 751-758.

Fantoni, I. e Lozano, R. (2000). Adaptive stabilization of underactuated flexible-joint robots using an energy approach and min-max algoritms, Proceedings of the American Control Conference June: 25112512 .

Ghorbel, F. e Spong, M. W. (2000). Integral manifolds of singularly perturbed systems with application to rigid-link flexible-joint multibody systems, International Journal of Non-linear Mechanics June(34): 133-155.

Guenther, R. (1993). Controle em cascata adaptativo e a estrutura variável de robôs Manipuladores com elos rígidos acionados eletricamente e elos rígidos e transmissões flexíveis, Tese de Doutorado - UFRJ, Brasil.

Guenther, R. e Hsu, L. (1993). Variable struture adaptative cascade control of rigid-link electrically driven robot manipulators, In Proc. of IEEE Conf. on Decision and Control .

Kokotovic, P. (1991). Joy of feedback: non linear and adaptive, In Proc. IEEE Conference on Decision and Control .

Krzysztof, P. J. e ElMaraghy, H. A. (1992). Dynamic decoupling for hybrid control of rigid/flexible joint robots interacting with the enviroment, IEEE Transactions on Robotics and Automation 8(5): 519534 .

Nicosia, S. e Tomei, P. (1988). On the feedback linearization of robots with elasctic joints, In Proc. IEEE Int. Conf. on Robotics and Automation pp. 180185.
Nicosia, S. e Tomei, P. (1992). A method to design adaptive controllers for flexible joint robots, In Proc. IEEE Int. Conf. on Robotics and Automation 10: 835-846.

Ramirez, A. R. G., De Pieri, E. e Guenther, R. (2002). Protótipo de robô planar projetado para o estudo da flexibilidade nas transmissões, Congresso Brasileiro de Automatica Setembro: 2138-2143.

Readman (1994). Flexible Joint Robots, CRC Press, Florida.

Rocco, P., Ferretti, G. e Magnani, G. (1996). Implicit force control for industrial robots in contact with stiff surfaces, IFAC World Congress 1b-11(2): 355360 .

Seibert, P. e Suarez, P. (1987). Global stabilization of cascade systems, System and Control Letters 14: $347-352$.

Slotine, J. J. E. e Li, W. (1991). Applied Nonlinear Control, Prentice Hall International.

Spong, M. W. (1987). Modelling and control of elastics joint robots, ASME, Journal of Dyn Syst. Meas. and Control 109: 310-319.

Spong, M. W. e Vidyasagar, M. (1989). Robot Dynamics and Control, Prentice Hall, Inc., New Jersey.

Tomei, P. (1991). A simple pd controller for robots with elastic joints, IEEE Transactions on Automatic Control 36(10): 1208-1213.

Youcef-Toumi, K. e Gutz, D. A. (1994). Impact and force control: Modelling and experiments, Journal of Dynamics Systems, Measurement and Control 116: $89-98$. 